\title{
circEIF4G2 modulates the malignant features of cervical cancer via the miR-218/HOXA1 pathway
}

\author{
YIFAN MAO $^{1}$, LIYA ZHANG ${ }^{2}$ and YUAN LI $^{2}$ \\ ${ }^{1}$ Department of Gynecology, The Second People's Hospital of Wuhu; ${ }^{2}$ Department of Geriatrics, \\ The First Affiliated Hospital of Wannan Medical College, Wuhu, Anhui 241000, P.R. China
}

Received June 25, 2018; Accepted March 6, 2019

DOI: $10.3892 / \mathrm{mmr} .2019 .10032$

\begin{abstract}
Circular RNAs (circRNAs) serve important roles in tumorigenesis and may be used as novel molecular biomarkers for clinical diagnosis. However, the role and molecular mechanisms of circRNAs in cervical cancer (CC) remain unknown. In the present study, circRNA isoform of eukaryotic translation initiation factor $4 \gamma 2$ (circEIF4G2) was revealed to be significantly upregulated in $\mathrm{CC}$ tissues and cell lines. Furthermore, increased expression of circEIF4G2 was associated with poor prognosis in patients with CC. circEIF4G2 knockdown suppressed the malignant features of CC cells, including cell proliferation, colony formation, migration and invasion. Additionally, circEIF4G2 was identified to serve as a sponge for microRNA-218 (miR-218), which targeted homeobox A1 (HOXA1). Furthermore, circEIF4G2 may increase the expression levels of HOXA1 by sponging miR-218. Rescue experiments suggested that transfection with a miR-218 inhibitor attenuated the inhibitory effects of circEIF4G2 knockdown on cell proliferation, migration and invasion. Furthermore, silencing HOXA1 reversed the effects of the miR-218 inhibitor on CC cells. Collectively, the present findings suggested that circEIF4G2 promoted cell proliferation and migration via the miR-218/HOXA1 pathway.
\end{abstract}

\section{Introduction}

Cervical cancer (CC) is a common gynecological malignant tumor that may be caused by various factors, including human papillomavirus infection, and genetic and epigenetic alterations (1). Recently, there has been an increase in the tendency of $\mathrm{CC}$, particularly in younger patients (2). Despite advances in the prevention and diagnosis of $\mathrm{CC}$ due to widespread screening,

Correspondence to: Professor Yuan Li, Department of Geriatrics, The First Affiliated Hospital of Wannan Medical College, 2 Zheshanxi Road, Wuhu, Anhui 241000, P.R. China

E-mail: liyuanahwh@126.com

Key words: cervical cancer, circular RNA isoform of eukaryotic translation initiation factor $4 \gamma 2$, microRNA-218, homeobox A1, proliferation a large number of patients are still diagnosed with invasive CC (3). Therefore, the identification of novel biomarkers and elucidation of the molecular mechanisms underlying $\mathrm{CC}$ are required for improving the early diagnosis and treatment of CC.

Circular RNAs (circRNAs) derive from back-spliced exons that form a covalently closed loop without 5 '-3' polarity or a polyadenylated tail (4). Advances in high-throughput DNA sequencing techniques have facilitated identification of the functions of various circRNAs. Accumulating evidence has demonstrated that circRNAs may be involved in cell cycle progression, cell aging and apoptosis by regulating gene expression and directly binding multiple proteins, thus affecting their activity $(5,6)$. Additionally, circRNAs serve important roles in tumorigenesis and have been identified as novel molecular biomarkers for the diagnosis of various diseases. In addition, the ability of circRNAs to serve as microRNA (miRNA/miR) sponges has been reported (7). circRNAs may compete with miRNAs for binding the 3 ' untranslated regions (3'-UTRs) of mRNAs, thus regulating gene expression. circRNA_0084043 has been identified to promote melanoma cell proliferation and migration via the miR-153-3p/snail family transcriptional repressor pathway (8). Li et al (9) demonstrated that the circRNA isoform of fibroblast growth factor receptor 4 promotes myoblast differentiation by regulating the expression levels of miR-107 and Wnt family member 3A. In addition, hsa_circ_0008039 has been reported to modulate the malignant characteristics of breast cancer by regulating the miR-432-5p/E2F transcription factor 3 axis (10). However, the role and the molecular mechanisms of circRNAs in CC progression remain unknown.

In the present study, circRNA isoform of eukaryotic translation initiation factor $4 \gamma 2$ (circEIF4G2; circbase ID: hsa_circ_0021254; www.circbase.org) expression was revealed to be increased in CC tissues, and upregulation of circEIF4G2 was associated with poor prognosis in patients with CC. Knockdown of circEIF4G2 suppressed proliferation and malignant features of CC cells. Mechanistically, the present findings suggested that circEIF4G2 may promote $\mathrm{CC}$ cell growth and migration by sponging miR-218, which decreased the expression levels of homeobox A1 (HOXA1).

\section{Patients and methods}

Patient samples. A total of 20 pairs of $\mathrm{CC}$ tissues and adjacent normal tissues were used in the present study. The samples 
were collected from patients with $\mathrm{CC}$ at the Second People's Hospital of Wuhu (Wuhu, China) from January 2015 to June 2017. Patients were recruited according to the following criteria: i) Patients were diagnosed and confirmed by histopathological examination; ii) patients did not receive systemic chemotherapy or radiotherapy in the pelvic cavity prior to surgery; and iii) follow-up data could be obtained from all patients. Patients with serious cardiovascular disease or other malignancies were excluded. Tumors were graded according to the tumor-node-metastasis (TNM) system (7th edition) maintained by the American Joint Committee on Cancer and the International Union for Cancer Control (11). Patients with $\mathrm{CC}$ were divided into high and low circEIF4G2 expression groups according to the median level of circEIF4G2 (cut-off value, 2.79). All patients provided written informed consent, and the present study was approved by the Clinical Research Ethics Committee of the Second People's Hospital of Wuhu. Following surgical resection, all tissues were snap-frozen in liquid nitrogen and stored at $-80^{\circ} \mathrm{C}$ until further analysis.

Cell lines and transfection. Human CC cell lines, including HeLa, CasKi, C33A and SiHa cells, were obtained from the American Type Culture Collection (Manassas, VA, USA). Control cells were derived from normal cervical tissues from patients that underwent hysterectomy. Briefly, normal cervical tissues were digested by $20 \%$ collagenase type $\mathrm{I}$ at $37^{\circ} \mathrm{C}$ for $40 \mathrm{~min}$ to obtain cervical epithelial cells. Dulbecco's Modified Eagle's medium (DMEM) supplemented with $10 \%$ fetal bovine serum (FBS; Gibco; Thermo Fisher Scientific, Inc., Waltham, MA, USA), L-glutamine (2 mM), penicillin (100 U/ml) and streptomycin $(100 \mathrm{mg} / \mathrm{ml}$; Sigma-Aldrich; Merck KGaA, Darmstadt, Germany) were used to culture CC cells and the control cells at $37^{\circ} \mathrm{C}$ with $5 \% \mathrm{CO}_{2}$.

Small interfering RNAs (siRNAs) targeting circEIF4G2 and HOXA1, siRNA-negative control (si-NC), miR-218 mimic, miR-NC, miR-218 inhibitor and miR-218 inhibitor-NC were purchased from Guangzhou RiboBio Co., Ltd. (Guangzhou, China). The sequences were as follows: si-circEIF4G2, 5'-AUG CUCCCAGCUUUUGGAAAA-3'; si-HOXA1, 5'-GGAUGU CUGUAAUAAAUAAAU-3'; si-NC, 5'-UUCUCCGAACGU GUCACGU-3'; miR-218 mimic, 5'-UUGUGCUUGAUCUAA CCAUGU-3'; miR-NC, 5'-UUCUCCGAACGUGUCACG U-3'; miR-218 inhibitor, 5'-ACAUGGUUAGAUCAAGCA CAA-3'; and miR-218 inhibitor-NC, 5'-CAGUACUUUUGU GUAGUACAA-3'. The cDNA sequence of circEIF4G2 was synthesized and cloned into the lentiviral expression vector pLVXIRES-neo (Clontech Laboratories, Inc., Mountainview, CA, USA) by Guangzhou RiboBio Co., Ltd., and the resulting p-circEIF4G2 vector ( $\left.1 \times 10^{9} \mathrm{PFU}\right)$ was used to overexpress circEIF4G2. 293T cells (Sangon Biotech Co., Ltd., Shanghai, China) were used for packaging the lentivirus. HeLa or C33A cells $\left(2 \times 10^{5}\right)$ were transfected with a combination of nucleic acids $(50 \mathrm{nM})$ using Lipofectamine ${ }^{\circledR} 3000$ (Invitrogen; Thermo Fisher Scientific, Inc.) according to the manufacturer's protocol. Following incubation for $48 \mathrm{~h}$, cells were harvested for further experimentation.

Cell Counting kit-8 (CCK-8) assay. HeLa or C33A cells in the logarithmic growth phase were cultured in 96-well plates $\left(1 \times 10^{4}\right.$ cells/well) overnight. Once cells reached
$60 \%$ confluence they were transfected and were then cultured for $24,48,72$ or $96 \mathrm{~h}$. At each time point, $10 \mu \mathrm{l} \mathrm{CCK-8} \mathrm{solution}$ (Dojindo Molecular Technologies, Inc., Kumamoto, Japan) was added for $4 \mathrm{~h}$. Cell proliferation was measured based on the absorbance detected at $450 \mathrm{~nm}$ using a microplate reader (Thermo Fisher Scientific, Inc.).

Colony formation assay. HeLa or C33A cells transfected with si-circEIF4G2 or si-NC were digested into single cells using trypsin. Cells $\left(5 \times 10^{2}\right.$ cells/well) were then mixed with $0.35 \%$ agarose and DMEM, and plated onto a solid layer of agarose/DMEM. Following solidification of the upper agarose layer, the plates were incubated at $37^{\circ} \mathrm{C}$ with $5 \% \mathrm{CO}_{2}$ for 2 weeks. Then, the colonies were fixed with $4 \%$ paraformaldehyde for $15 \mathrm{~min}$ and stained with $0.05 \%$ crystal violet for $30 \mathrm{~min}$ at room temperature. The number of colonies was assessed using a light microscope (magnification, x100).

Wound healing assay. HeLa or C33A cells were seeded in 6 -well plates at a density of $5 \times 10^{5}$ cells/well. The cells were then scratched in a straight line using a $10-\mu 1$ sterile pipette tip and the cellular debris was washed using PBS. Subsequently, the cells were incubated at $37^{\circ} \mathrm{C}$ for $48 \mathrm{~h}$ and images of the wounds were captured using an IX71 microscope (magnification, x100; Olympus Corporation, Tokyo, Japan).

Matrigel invasion assay. Transfected HeLa or C33A cells $\left(2.5 \times 10^{3}\right.$ cells/well) in serum-free DMEM were plated in the upper chamber of a Transwell system with $8-\mu \mathrm{m}$ pores, which was pre-coated with Matrigel (BD Biosciences, Franklin Lakes, NJ, USA). DMEM containing 10\% FBS was added to the lower chamber. After a 24-h incubation, the invasive cells were fixed with methanol at $37^{\circ} \mathrm{C}$ for $30 \mathrm{~min}$ and stained with $0.1 \%$ crystal violet at $37^{\circ} \mathrm{C}$ for $30 \mathrm{~min}$. Subsequently, images of the invasive cells were captured and the cells were counted using an IX71 microscope (magnification, x100).

Reverse transcription-quantitative polymerase chain reaction $(R T-q P C R)$. Total RNA was isolated from tissues or cells using TRIzol ${ }^{\circledR}$ reagent (Invitrogen; Thermo Fisher Scientific, Inc.). Following quantification using a NanoDrop ${ }^{\mathrm{TM}}$ spectrophotometer (NanoDrop Technologies; Thermo Fisher Scientific, Inc., Wilmington, DE, USA), RNA underwent cDNA synthesis using a High Capacity cDNA RT kit (Applied Biosystems; Thermo Fisher Scientific, Inc., Waltham, MA, USA) according to the manufacturer's protocol. qPCR was performed using the SYBR Select Master Mix (Applied Biosystems; Thermo Fisher Scientific, Inc.) on an ABI 7900 system (Applied Biosystems; Thermo Fisher Scientific, Inc.), with GAPDH or U6 as an internal control. The PCR conditions were as follows: $95^{\circ} \mathrm{C}$ for $3 \mathrm{~min}$, followed by 40 cycles of $95^{\circ} \mathrm{C}$ for $10 \mathrm{sec}, 60^{\circ} \mathrm{C}$ for $30 \mathrm{sec}$ and $72^{\circ} \mathrm{C}$ for $30 \mathrm{sec}$. Primer sequences are listed in Table I. The $2^{-\Delta \Delta \mathrm{Cq}}$ method was used to determine the relative gene expression level (12).

Western blot analysis. Total protein was extracted from transfected HeLa or C33A cells lysed using the Pierce cell lysis buffer (Pierce; Thermo Fisher Scientific, Inc., Waltham, MA, USA). Bicinchoninic acid protein assay kit (Takara Biotechnology Co., Ltd., Dalian, China) was used to quantify 
Table I. Primers used for reverse transcription-quantitative polymerase chain reaction.

\begin{tabular}{lll}
\hline Gene symbol & \multicolumn{1}{c}{ Forward primer $\left(5^{\prime} \rightarrow 3^{\prime}\right)$} & Reverse primer $\left(5^{\prime} \rightarrow 3^{\prime}\right)$ \\
\hline circEIF4G2 & TTTTTCAACAAAGCAAGGTCAA & TCTAGGTCCCACTGTCCTCA \\
miR-218 & CGCGCGCGTTGTGCTTGATCTAA & AGTGCAGGGTCCGAGGTATT \\
HOXA1 & GGGTGTCCTACTCCCACTCA & GGACCATGGGAGATGAGAGA \\
GAPDH & GTGTTTCCTCGTCCCGTAGA & GAATTTGCCGTGAGTGGAGT \\
U6 & GCTTCGGCAGCACATATACTAAAAT & TACTGTGCGTTTAAGCACTTCGC
\end{tabular}

circEIF4G2, circular RNA isoform of eukaryotic translation initiation factor 4 $\gamma 2$; HOXA1, homeobox A1; miR, microRNA.

protein concentration. Equal amounts of protein $(30 \mu \mathrm{g} / \mathrm{lane})$ were separated by $10 \%$ SDS-PAGE and were subsequently transferred onto polyvinylidene difluoride membranes (EMD Millipore, Billerica, MA, USA). The membranes were blocked in $5 \%$ skim milk for $2 \mathrm{~h}$ at $37^{\circ} \mathrm{C}$, and then incubated with primary antibodies overnight at $4^{\circ} \mathrm{C}$. The primary antibodies used were anti-HOXA1 (1:1,000; cat. no. ab230513) and anti- $\beta$-actin (1:2,000; cat. no. ab8227; Abcam, Cambridge, UK). Subsequently, membranes were incubated for $2 \mathrm{~h}$ at room temperature with horseradish peroxidase-conjugated secondary antibody (1:2,000; cat. no. ab97051; Abcam). Bands were visualized using an enhanced chemiluminescence detection kit (EMD Millipore).

Target prediction. The potential miRNAs interacting with circEIF4G2 were predicted using Starbase 2.0 (http://starbase. sysu.edu.cn/). Putative targets of miR-218 were predicted using TargetScan (release 6.2; http://www.targetscan. org), microRNA.org (version 3.3a; http://www.microrna. org/microrna/home.do) and miRDB (http://www.mirdb.org).

Dual-luciferase reporter assay. To construct the recombinant luciferase vectors, wild type (WT) and mutant (MUT) circEIF4G2 sequences were synthesized by Shanghai GenePharma Co., Ltd. (Shanghai, China) and separately cloned into pmirGLO luciferase vectors (Promega Corporation, Madison, WI, USA) between the NheI and $\mathrm{XbaI}$ sites. In addition, WT and MUT 3'-UTRs of HOXA1 were synthesized by Shanghai GenePharma Co., Ltd. and separately cloned into pmirGLO luciferase vectors between the SacI and XbaI sites. HeLa or C33A cells were cotransfected with $50 \mathrm{nM}$ miR-218 mimic or miR-NC, and the recombinant luciferase vectors using Lipofectamine ${ }^{\circledR} 3000$ (Invitrogen; Thermo Fisher Scientific, Inc.). After a 48-h incubation, luciferase activity was detected using a dual-luciferase reporter assay system (Promega Corporation) according to the manufacturer's protocol. Renilla luciferase activity was detected and used as the internal control.

RNA immunoprecipitation (RIP) assay. RNA-binding protein immunoprecipitation kit (EMD Millipore) was used to perform the RIP assay according to the manufacturer's protocol. HeLa or C33A cells at 80-90\% confluency were incubated with the RIP buffer containing magnetic beads coated with anti-argonaute RNA-induced silencing complex (RISC) catalytic component 2 (AGO2) antibodies (cat. no. MABE253, EMD Millipore) or anti-immunoglobulin G (IgG; cat. no. AB22-K, EMD Millipore) antibodies. RT-qPCR was performed to detect the expression levels of immunoprecipitated RNA of circEIF4G2 and miR-218.

Statistical analysis. Data were analyzed using SPSS 17.0 software (SPSS, Inc., Chicago, IL, USA). Data are presented as the means \pm standard deviation from three experiments. Comparisons between two groups were analyzed using Student's t-test, whereas multiple groups were compared using one-way analysis of variance followed by Tukey's post hoc test. Kaplan-Meier analysis was performed to calculate the overall survival rate, and a log-rank test was conducted to compare the survival distributions between two groups. Pearson correlation analysis was performed between the expression levels of circEIF4G2 and miR-218 in CC tissues. $\mathrm{P}<0.05$ was considered to indicate a statistically significant difference.

\section{Results}

circEIF4G2 is upregulated in CC tissues and cells. To investigate the expression levels of circEIF4G2 in CC, 20 pairs of $\mathrm{CC}$ tissues and corresponding adjacent normal tissues were collected and RT-qPCR was performed to detect circEIF4G2 expression. The clinical characteristics of patients with $\mathrm{CC}$ are presented in Table II. High expression of circEIF4G2 was significantly associated with tumor size and lymph node metastasis, but not with age or tumor-node-metastasis stage (Table II). circEIF4G2 was significantly upregulated in $\mathrm{CC}$ tissues compared with in adjacent normal tissues (Fig. 1A). Increased expression of circEIF4G2 was also detected in various CC cell lines, including HeLa, CasKi, C33A and $\mathrm{SiHa}$ cells, compared with in control cells derived from tissues from patients that underwent hysterectomy (Fig. 1B). To examine the possibility of using circEIF4G2 expression as a prognostic marker in patients with CC, Kaplan-Meier curve analysis was performed, and $\mathrm{CC}$ samples exhibiting an increased expression of circEIF4G2 were compared with $\mathrm{CC}$ samples exhibiting a decreased expression level of circEIF4G2. The results suggested that increased expression levels of circEIF4G2 were associated with poor prognosis in patients with CC (Fig. 1C).

circEIF4G2 knockdown suppresses the proliferation and malignant features of CC cells. To investigate the role of circEIF4G2 in CC cells, HeLa and C33A cells were selected for further analyses, due to the increased expression level of circEIF4G2. A siRNA targeting circEIF4G2 was designed and transfected 
Table II. Clinical characteristics of patients with cervical cancer.

Expression level of circEIF4G2

relative to the median

Clinicopathological characteristic

Low, $\mathrm{n}=10$

High, $\mathrm{n}=10$

P-value

Age

0.370

$\leq 45$ years

4

$>45$ years

6

7

Tumor size

$\leq 4 \mathrm{~cm}$

7

$>4 \mathrm{~cm}$

Lymph node metastasis

$0.011^{\mathrm{a}}$

Negative

6

Positive

TNM stage

I/II

0

10

III/IV

4

6

${ }^{\mathrm{a}} \mathrm{P}<0.05$. circEIF4G2, circular RNA isoform of eukaryotic translation initiation factor $4 \gamma 2$; TNM, tumor-node-metastasis.

A

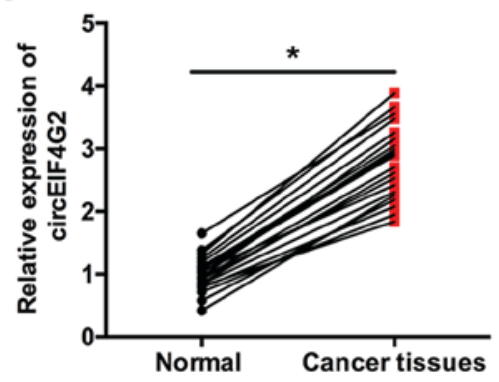

B

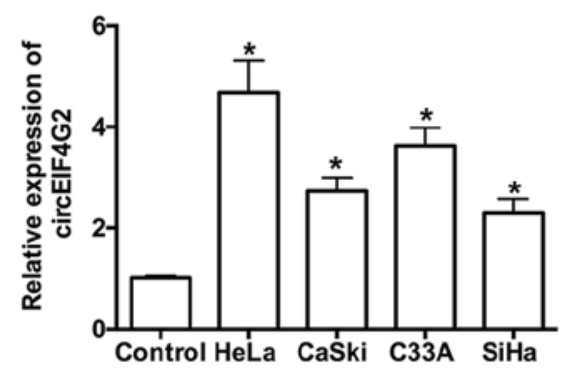

C

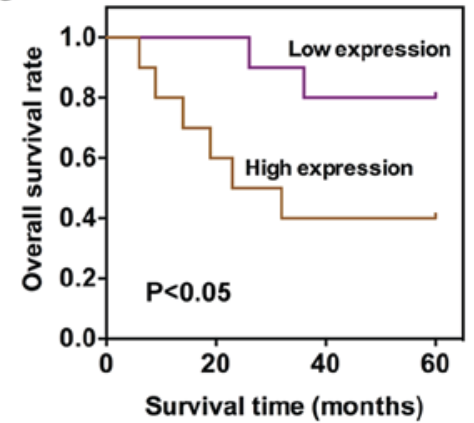

Figure 1. circEIF4G2 is upregulated in CC tissues and cell lines. Reverse transcription-quantitative polymerase chain reaction results of the relative expression level of circEIF4G2 in (A) 20 pairs of CC tissues and adjacent normal tissues and in (B) CC cell lines. Adjacent normal tissues from patients who underwent hysterectomy, and cells derived from these tissues, were used as the respective controls. (C) Kaplan-Meier analysis of the association between circEIF4G2 expression and survival. ${ }^{*} \mathrm{P}<0.05$ vs. corresponding control. CC, cervical cancer; circEIF4G2, circular RNA isoform of eukaryotic translation initiation factor $4 \gamma 2$.

into HeLa and C33A cells. The RT-qPCR analysis revealed that following transfection with si-circEIF4G2, the expression levels of circEIF4G2 were decreased by $62.3 \%$ in HeLa cells and by $61.3 \%$ in $\mathrm{C} 33 \mathrm{~A}$ cells compared with the corresponding control (Fig. 2A). Subsequently, CCK-8 assay and colony formation assay were performed to examine the effects of circEIF4G2 knockdown on cell proliferation. circEIF4G2 knockdown significantly suppressed cell proliferation and colony formation in HeLa and C33A cells (Fig. 2B-D). Wound healing and Matrigel invasion assays were performed to investigate the role of circEIF4G2 in the malignant features of HeLa and C33A cells. The results suggested that circEIF4G2 knockdown significantly inhibited cell migration and invasion (Fig. 2E and F).

circEIF4G2 inhibits the expression of miR-218. To investigate the mechanism underlying circEIF4G2 function, the potential miRNAs interacting with circEIF4G2 were predicted using
Starbase 2.0, as circRNAs may serve as sponges of miRNAs, thus regulating gene expression. The in silico results suggested that a possible miR-218 binding site was present in the sequence of circEIF4G2 (Fig. 3A). The expression levels of miR-218 were upregulated following transfection with miR-218 mimic, conversely, transfection with miR-218 inhibitor downregulated the expression levels of miR-218 in HeLa and C33A cells (Fig. 3B). Furthermore, dual-luciferase reporter assay results suggested that miR-218 overexpression significantly suppressed the luciferase activity of WT-circEIF4G2 luciferase plasmid; however, the luciferase activity of the plasmid containing the MUT-circEIF4G2 sequence was not affected in HeLa or C33A cells (Fig. 3C). To examine the role of circEIF4G2 on miR-218 expression, RT-qPCR was performed following circEIF4G2 knockdown. The results suggested that the expression levels of miR-218 in HeLa and C33A cells transfected with si-circEIF4G2 exhibited a 4.97- and 3.70-fold 
A

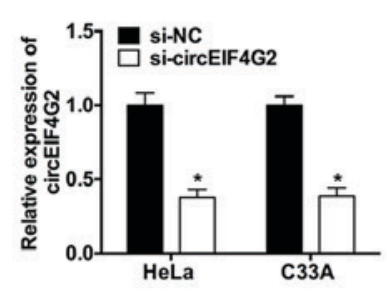

E

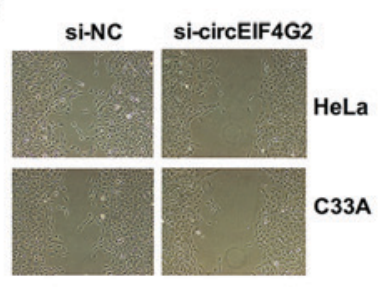

B
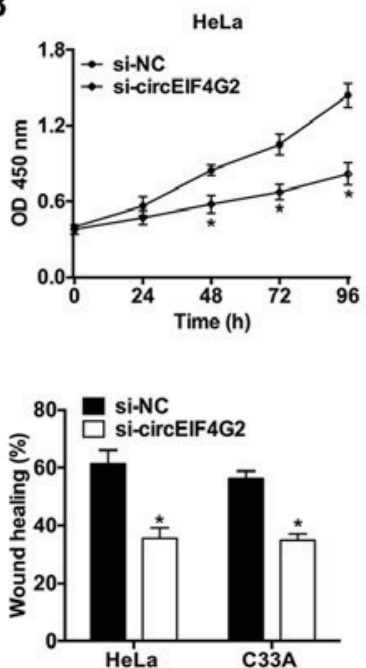

C

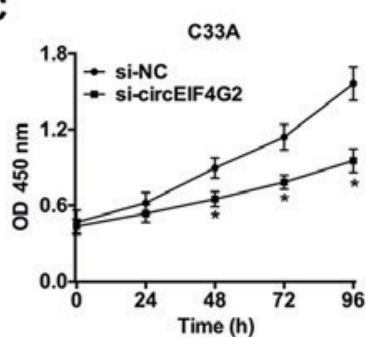

$\mathbf{F}$

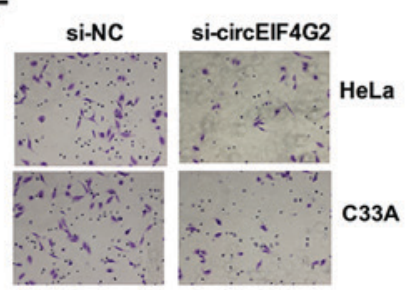

D

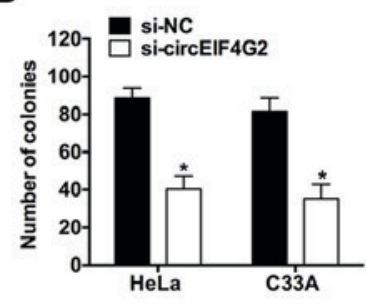

Figure 2. circEIF4G2 knockdown suppresses proliferation and the malignant features of $\mathrm{CC}$ cells. (A) Expression levels of circEIF4G2 following transfection with si-circEIF4G2. Cell Counting kit-8 assay was performed to detect proliferation of (B) HeLa and (C) C33A cells transfected with si-circEIF4G2 or si-NC. (D) Colony formation assay was performed in HeLa and C33A cells transfected with si-circEIF4G2 or si-NC. (E) Wound healing (magnification, x100) and (F) invasion (magnification, $\mathrm{x} 100$ ) assay results of HeLa and C33A cells following transfection with si-circEIF4G2 or si-NC. "P $<0.05$ vs. si-NC group. $\mathrm{CC}$, cervical cancer; circEIF4G2, circular RNA isoform of eukaryotic translation initiation factor $4 \gamma 2$; NC, negative control; OD, optical density; si-, small interfering RNA.

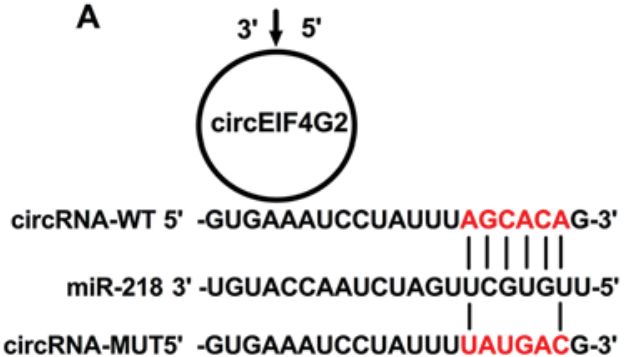

D

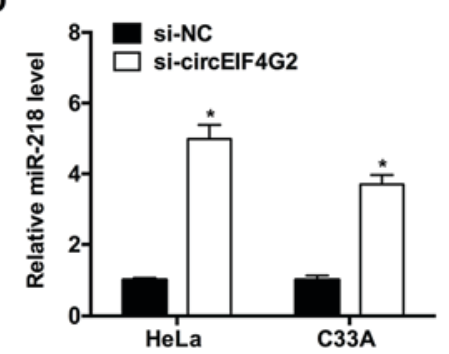

G

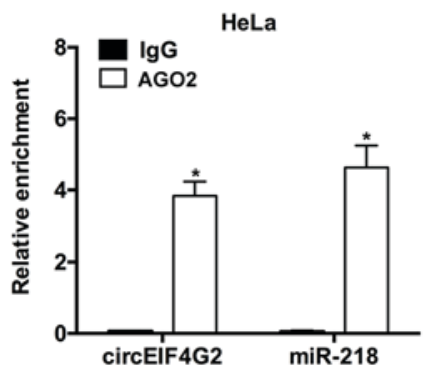

B
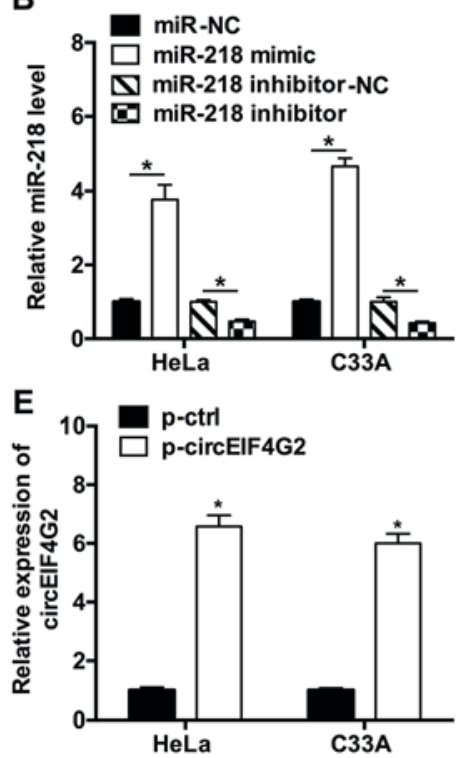

H

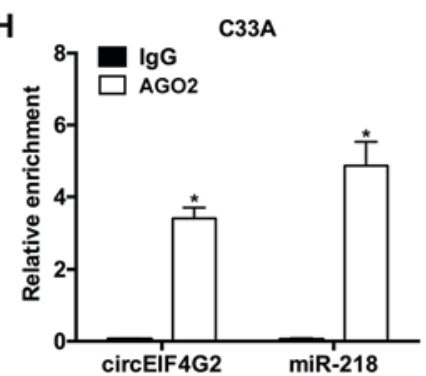

C

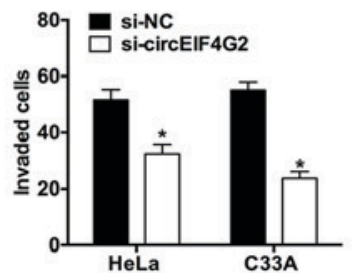


A

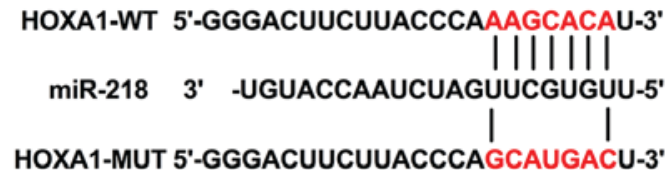

HOXA1-MUT 5'-GGGACUUCUUACCCAGCAUGACU-3'
B

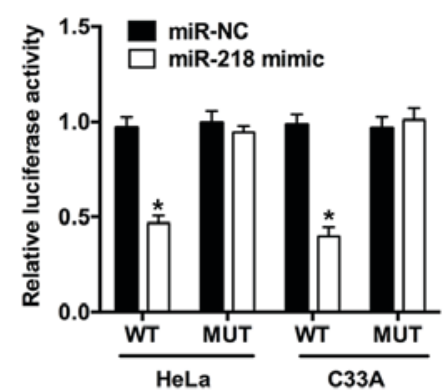

$c$

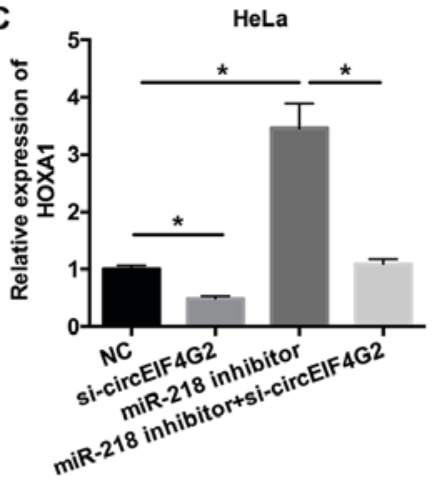

D

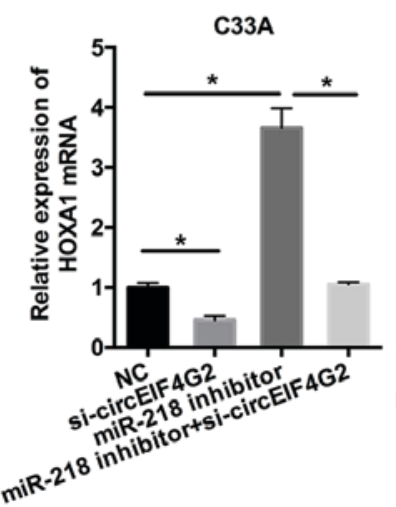

E

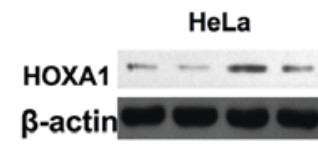

C33A

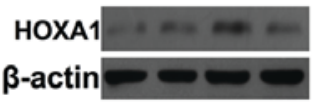

$\mathrm{NC}+--$

si-circEIF4G2 - + -+

miR-218 inhibitor $-\quad++$
$\mathbf{F}$

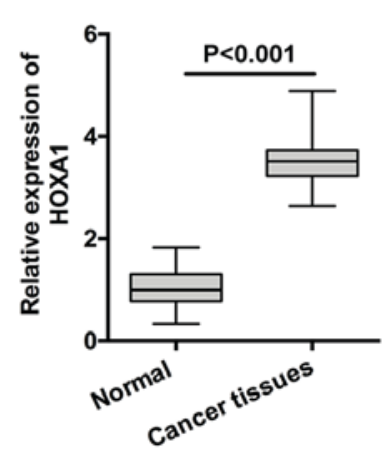

G

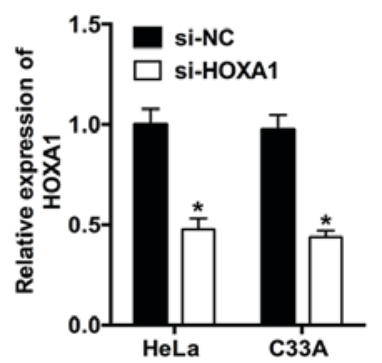

Figure 4. circEIF4G2 increases HOXA1 expression by sponging miR-218. (A) Predicted interaction between miR-218 and the 3'-UTR of HOXA1. (B) Interaction between miR-218 and HOXA1 3'-UTR was examined in HeLa and C33A cells by dual-luciferase reporter assay. Expression levels of HOXA1 in (C) HeLa and (D) C33A cells transfected with si-circEIF4G2 and/or miR-218 inhibitor. (E) Protein expression levels of HOXA1 in HeLa and C33A cells transfected with si-circEIF4G2 and/or miR-218 inhibitor. (F) Expression levels of HOXA1 in CC tissues. (G) Expression levels of HOXA1 in CC cells transfected with si-HOXA1 or si-NC. ${ }^{*} \mathrm{P}<0.05$ vs. corresponding control. circEIF4G2, circular RNA isoform of eukaryotic translation initiation factor $4 \gamma 2$; HOXA1, homeobox A1; miR, microRNA; MUT, mutant; NC, negative control; si-, small interfering RNA; UTR, untranslated region; WT, wild type.

increase compared with HeLa and C33A cells transfected with si-NC, respectively (Fig. 3D). The expression levels of circEIF4G2 were significantly increased in HeLa and C3AA cells transfected with p-circEIF4G2 (Fig. 3E). Additionally, miR-218 expression levels were significantly downregulated in HeLa and C33A cells transfected with p-circEIF4G2 (Fig. 3F). RIP assay was performed in HeLa and C33A cells using anti-AGO2 antibodies, and the expression levels of circEIF4G2 and miR-218 were detected using RT-qPCR. The RIP results suggested that circEIF4G2 and miR-218 were significantly enriched following immunoprecipitation of AGO2 compared with IgG (Fig. 3G and H). Furthermore, the correlation analysis of the expression levels of circEIF4G2 and miR-218 suggested that the expression levels of miR-218 and circEIF4G2 were negatively correlated in CC tissues (Fig. 3I).

circEIF4G2 induces the expression of HOXAl by sponging $m i R-218$. The downstream mechanism of the circEIF4G2/miR-218 axis was further investigated. Following in silico prediction analysis, HOXA1 was identified to be a potential target of miR-218 (Fig. 4A). To examine the association between miR-218 and HOXA1, dual-luciferase reporter assay was performed and the data suggested that transfection with miR-218 mimic significantly suppressed the luciferase activity of HOXA1-WT plasmid compared with the negative control (Fig. 4B); however, the luciferase activity of HOXA1-MUT plasmid was not affected. To further investigate the effects of miR-218 and circEIF4G 2 on HOXA1 expression, HeLa and C33A cells were transfected with si-circEIF4G2 and/or miR-218 inhibitor. Knockdown of circEIF4G2 significantly decreased the expression levels of HOXA1, whereas transfection with miR-218 inhibitor increased the expression levels of HOXA1 (Fig. 4C and D). The results revealed that, compared with cells transfected with miR-218 inhibitor, the concomitant knockdown of circEIF4G2 and miR-218 decreased the expression levels of HOXA1 by 68.5 and $71.1 \%$ in HeLa cells and C33A cells, respectively (Fig. 4C and D). The protein expression levels of HOXA1 were consistent with the RT-qPCR results (Fig. 4E). The mRNA expression levels of HOXA1 in CC tissues were analyzed and HOXA1 was identified to be upregulated in $\mathrm{CC}$ tissues compared with in normal tissues (Fig. 4F). In addition, the expression levels of HOXA1 were significantly decreased following transfection with si-HOXA1 in HeLa or C33A cells (Fig. 4G). Therefore, si-HOXA1 was used to knockdown HOXA1 in the further experiments.

circEIF4G2 promotes cell proliferation and migration via the miR-218/HOXA1 pathway. To further investigate the role of the miR-218/HOXA1 axis in the oncogenic function of circEIF4G2, rescue experiments were performed in HeLa and C33A cells. Transfection with the miR-218 inhibitor attenuated the inhibitory effects of circEIF4G2 knockdown on proliferation, migration and invasion of HeLa and C33A cells (Fig. 5). 
A

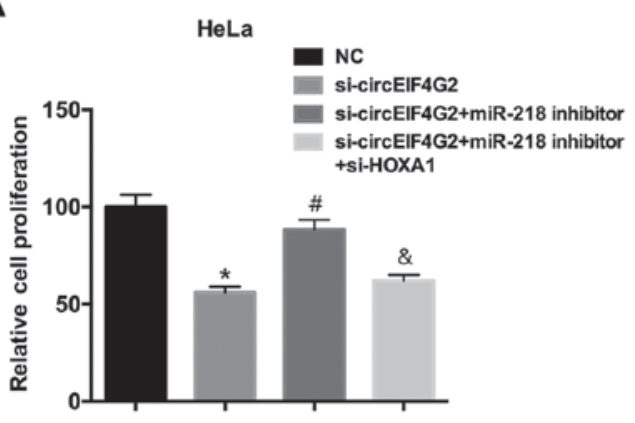

C

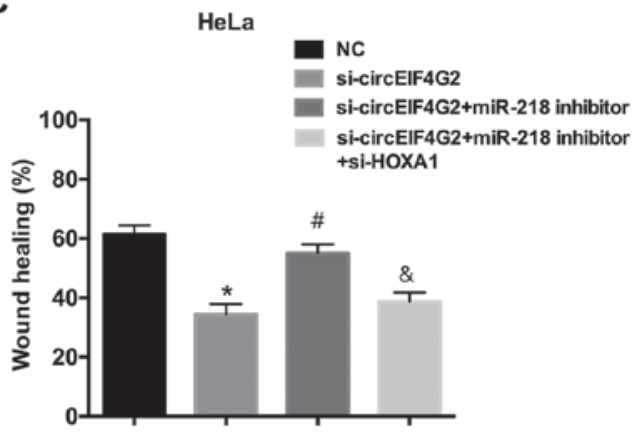

E

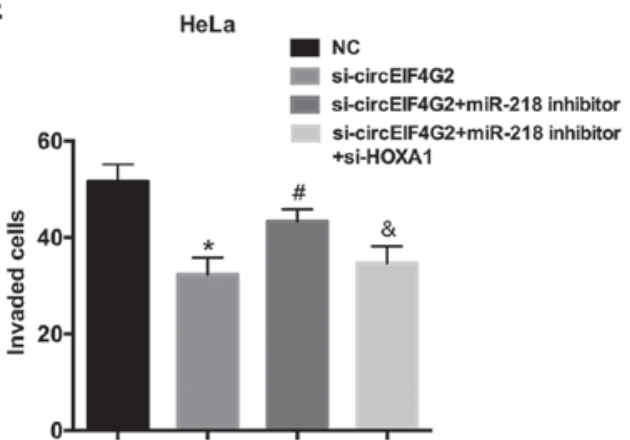

B

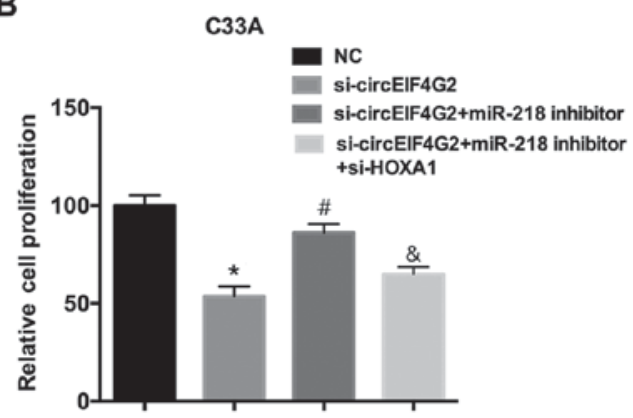

D

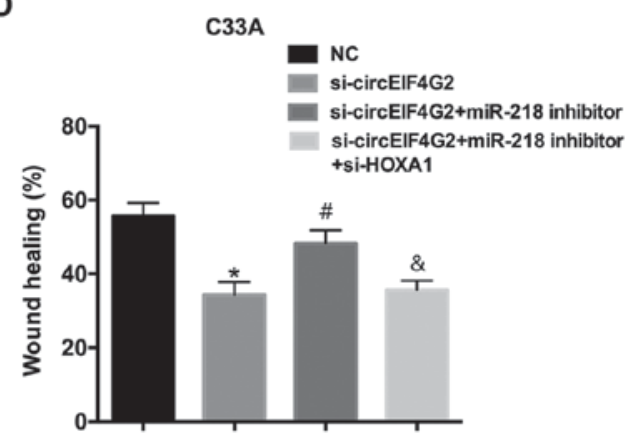

F

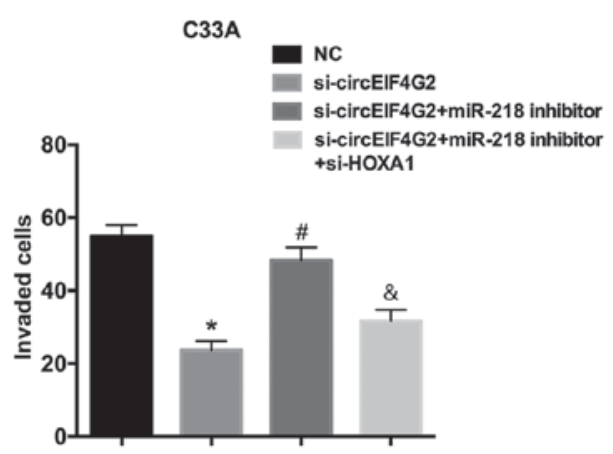

Figure 5. circEIF4G2 promotes cell proliferation and migration via the miR-218/HOXA1 pathway. HeLa cells and C33A cells were transfected with si-circEIF4G2, si-circEIF4G2 + miR-218 inhibitor or si-circEIF4G2 + miR-218 inhibitor + si-HOXA1. Proliferation of (A) HeLa and (B) C33A cells was detected by Cell Counting kit-8 assay. Migration of (C) HeLa and (D) C33A cells was assessed by wound healing assay. Invasion of (E) HeLa and (F) C33A cells was detected by Matrigel assay. ${ }^{*} \mathrm{P}<0.05$ vs. si-NC group, ${ }^{~} \mathrm{P}<0.05$ vs. si-circEIF4G2 group, ${ }^{\circledR} \mathrm{P}<0.05$ vs. si-circEIF4G2 + miR-218 inhibitor group. circEIF4G2, circular RNA isoform of eukaryotic translation initiation factor 4 $\gamma 2$; HOXA1, homeobox A1; miR-, microRNA; NC, negative control; si-, small interfering RNA.

Furthermore, HOXA1 knockdown reversed the effects of the miR-218 inhibitor. Collectively, the present data suggested that circEIF4G2 may promote cell proliferation and migration via the miR-218/HOXA1 pathway.

\section{Discussion}

Accumulating evidence has demonstrated that the dysregulation of circRNAs is associated with the occurrence and development of cancer and other diseases $(13,14)$. circRNAs have been identified to exhibit an increased stability compared with linear RNAs due to their closed-loop structure and resistance to RNase R (15). circRNAs are expressed in various human cells and certain circRNAs exhibit tissue- and disease-specificity. The expression levels of circRNAs have been reported to be increased in brain tissues, particularly in synapses. Due to the advances in circRNA microarray and bioinformatics analyses, the identity and function of numerous novel circRNAs have been identified. For example, Chen et al (16) demonstrated, using circRNA microarray analysis, that the expression levels of hsa_circ_0128298 are increased in hepatocellular carcinoma (HCC) tissues, and that hsa_circ_0128298 may be used as a novel diagnostic and prognostic biomarker for patients with HCC. Wang et al (17) demonstrated that circ_0067934 serves as an oncogene and its expression levels are associated with poor prognosis in patients with non-small cell lung cancer. In the present study, circEIF4G2 was identified to be upregulated in CC tissues and cells compared with in adjacent normal tissues and cells. Furthermore, an increase in the expression levels of circEIF4G2 was associated with poor prognosis in patients with CC. In CC cells, loss-of-function experiments suggested that circEIF4G2 knockdown suppressed cell proliferation, colony formation, migration and invasion, indicating that circEIF4G2 may serve oncogenic roles.

It has been demonstrated that circRNAs may serve as miRNA sponges. To investigate the molecular mechanism of 
circEIF4G2 in CC, the potential targets of circEIF4G2 were predicted via bioinformatics analysis and the results suggested that miR-218 may be a target of circEIF4G2. To validate the in silico prediction results, luciferase activity and RIP assays were performed; the in vitro results suggested that circEIF4G2 may interact with miR-218 in CC cells. The RIPresults suggested that circEIF4G2 was present in AGO2-containing RISCs via physical associations with miR-218. It has been reported that AGO2 is a core component of the RISC, the formation of which is a necessary step in RNA silencing; miRNAs were present in the cytoplasm in the form of miRNA-ribonucleoprotein complexes that also contained AGO2 (18). RT-qPCR results suggested that circEIF4G2 knockdown increased the expression levels of miR-218 in CC cells. Additionally, the expression levels of miR-218 and circEIF4G2 were negatively correlated in CC tissues. Our previous study demonstrated that miR-218 suppresses cell progression by targeting APC regulator of WNT signaling pathway in CC (19). miR-218 has previously been demonstrated to serve as a tumor suppressor in various types of cancer, including ovarian, bladder and prostate cancer (20-22). In the present study, transfection with the miR-218 inhibitor reversed the inhibitory effects of circEIF4G2 knockdown, and restored the malignant features of HeLa and C33A cells.

The present study further investigated the coding gene downstream to the circEIF4G2/miR-218 axis. HOXA1 was identified as a target of miR-218 in CC cells. Furthermore, it was suggested that circEIF4G2 increased the expression levels of HOXA1 by sponging miR-218. HOXA1 is a member of the HOXA gene family, which has previously been demonstrated to serve roles in organogenesis and in the regulation of cell fate (23). Numerous studies have reported that the dysregulation of HOXA1 is associated with human cancer progression, including colon cancer and esophageal cancer $(24,25)$. These previous studies observed that HOXA1 serves as an oncogene, which is able to promote cell growth, invasion and metastasis. A recent study by Eoh et al (26) demonstrated that upregulation of HOXA1 is associated with poor survival rate in patients with in $\mathrm{CC}$. In the present study, HOXA1 knockdown was able to reverse the effects of the miR-218 inhibitor on CC cells, and circEIF4G2 was able to promote cell proliferation and migration via the miR-218/HOXA1 pathway.

Collectively, the present results suggested that circEIF4G2 may be a novel oncogene in CC. Furthermore, circEIF4G2 may promote $\mathrm{CC}$ cell growth and migration by sponging miR-218, thus increasing the expression levels of HOXA1.

\section{Acknowledgements}

Not applicable.

\section{Funding}

The present study was supported by The National Science Foundation of China (grant no. 8742543).

\section{Availability of data and materials}

The datasets used and/or analyzed during the current study are available from the corresponding author on reasonable request.

\section{Authors' contributions}

YM and YL designed and conceived the present study, and performed the experiments. LZ analyzed the data.

\section{Ethics approval and consent to participate}

The present study was approved by the Clinical Research Ethics Committee of The Second People's Hospital of Wuhu. All patients provided written informed consent.

\section{Patient consent for publication}

Not applicable.

\section{Competing interests}

The authors declare that they have no competing interests.

\section{References}

1. Cordeiro MN, De Lima RCP, Paolini F, Melo ARDS, Campos APF, Venuti A and De Freitas AC: Current research into novel therapeutic vaccines against cervical cancer. Expert Rev Anticancer Ther 18: 365-376, 2018.

2. Castanon A and Sasieni P: Is the recent increase in cervical cancer in women aged 20-24 years in England a cause for concern? Prev Med 107: 21-28, 2018.

3. Origoni M, Prendiville W and Paraskevaidis E: Cervical cancer prevention: New frontiers of diagnostic strategies. Biomed Res Int 2015: 250917, 2015.

4. Chen LL and Yang L: Regulation of circRNA biogenesis. RNA Biol 12: 381-388, 2015

5. Salzman J: Circular RNA expression: Its potential regulation and function. Trends Genet 32: 309-316, 2016.

6. Ebbesen KK, Kjems J and Hansen TB: Circular RNAs: Identification, biogenesis and function. Biochim Biophys Acta 1859: 163-168, 2016.

7. Han B, Chao J and Yao H: Circular RNA and its mechanisms in disease:Fromthebenchtotheclinic.PharmacolTher 187:31-44,2018.

8. Luan W, Shi Y, Zhou Z, Xia Y and Wang J: circRNA 0084043 promote malignant melanoma progression via miR-153-3p/Snail axis. Biochem Biophys Res Commun 502: 22-29, 2018.

9. Li H, Wei X, Yang J, Dong D, Hao D, Huang Y, Lan X, Plath M, Lei C, Ma Y, et al: circFGFR4 promotes differentiation of myoblasts via binding miR-107 to relieve its inhibition of Wnt3a. Mol Ther Nucleic Acids 11: 272-283, 2018.

10. Liu Y, Lu C, Zhou Y, Zhang Z and Sun L: Circular RNA hsa_circ_0008039 promotes breast cancer cell proliferation and migration by regulating miR-432-5p/E2F3 axis. Biochem Biophys Res Commun 502: 358-363, 2018.

11. Edge SB and Compton CC: The American Joint Committee on Cancer: The 7th edition of the AJCC cancer staging manual and the future of TNM. Ann Surg Oncol 17: 1471-1474, 2010.

12. Livak KJ and Schmittgen TD: Analysis of relative gene expression data using real-time quantitative PCR and the 2(-Delta Delta C(T)) method. Methods 25: 402-408, 2001.

13. Dragomir M and Calin GA: Circular RNAs in cancer-lessons learned from microRNAs. Front Oncol 8: 179, 2018.

14. Li M, Ding W, Sun T, Tariq MA, Xu T, Li P and Wang J: Biogenesis of circular RNAs and their roles in cardiovascular development and pathology. FEBS J 285: 220-232, 2018.

15. Qu S, Yang X, Li X, Wang J, Gao Y, Shang R, Sun W, Dou K and Li H: Circular RNA: A new star of noncoding RNAs. Cancer Lett 365: 141-148, 2015.

16. Chen D, Zhang C, Lin J, Song X and Wang H: Screening differential circular RNA expression profiles reveal that hsa_circ_0128298 is a biomarker in the diagnosis and prognosis of hepatocellular carcinoma. Cancer Manag Res 10: 1275-1283, 2018.

17. Wang J and Li H: CircRNA circ_0067934 silencing inhibits the proliferation, migration and invasion of NSCLC cells and correlates with unfavorable prognosis in NSCLC. Eur Rev Med Pharmacol Sci 22: 3053-3060, 2018. 
18. Filipowicz W, Bhattacharyya SN and Sonenberg N: Mechanisms of post-transcriptional regulation by microRNAs: Are the answers in sight? Nat Rev Genet 9: 102-114, 2008.

19. Mao Y,Zhang L, Li Y, Yan M and He L: MiR-218 suppresses cell progression by targeting APC in cervical cancer. Int J Clin Exp Pathol 10: 2259-2269, 2017

20. Zhu W, Shao Y and Peng Y: MicroRNA-218 inhibits tumor growth and increases chemosensitivity to CDDP treatment by targeting BCAT1 in prostate cancer. Mol Carcinog 56: 1570-1577, 2017.

21. Li P, Yang X, Cheng Y, Zhang X, Yang C, Deng X, Li P, Tao J, Yang H, Wei J, et al: MicroRNA-218 increases the sensitivity of bladder cancer to cisplatin by targeting Glut1. Cell Physiol Biochem 41: 921-932, 2017.

22. Li N, Wang L, Tan G, Guo Z, Liu L, Yang M and He J: MicroRNA-218 inhibits proliferation and invasion in ovarian cancer by targeting Runx2. Oncotarget 8: 91530-91541, 2017.

23. De Kumar B, Parker HJ, Paulson A, Parrish ME, Zeitlinger J and Krumlauf R: Hoxa1 targets signaling pathways during neural differentiation of ES cells and mouse embryogenesis. Dev Biol 432: 151-164, 2017.
24. Li H, Li J, Yang T, Lin S and Li H: MicroRNA-433 represses proliferation and invasion of colon cancer cells by targeting homeobox A1. Oncol Res 26: 315-322, 2018.

25. Li Q, Zhang X, Li N, Liu Q and Chen D: miR-30b inhibits cancer cell growth, migration, and invasion by targeting homeobox A1 in esophageal cancer. Biochem Biophys Res Commun 485: 506-512, 2017.

26. Eoh KJ, Kim HJ, Lee JY, Nam EJ, Kim S, Kim SW and Kim YT: Upregulation of homeobox gene is correlated with poor survival outcomes in cervical cancer. Oncotarget 8: 84396-84402, 2017.

This work is licensed under a Creative Commons Attribution-NonCommercial-NoDerivatives 4.0 International (CC BY-NC-ND 4.0) License. 